\title{
Knocking out FLI1 to clear the AIRE
}

\begin{abstract}
A new mouse model has shed light on the autoimmune phenotype observed in systemic sclerosis (SSc) and the unexplained tissue specificity of this disease. In this mouse model, conditional deletion of the transcription factor FLI1 in epithelial cells recapitulates the disease phenotype observed in patients with SSc. "Our findings seemed to prove the notion that 'global' epithelial abnormality plays a fundamental role in this disease," reports corresponding author Yoshihide Asano.
\end{abstract}

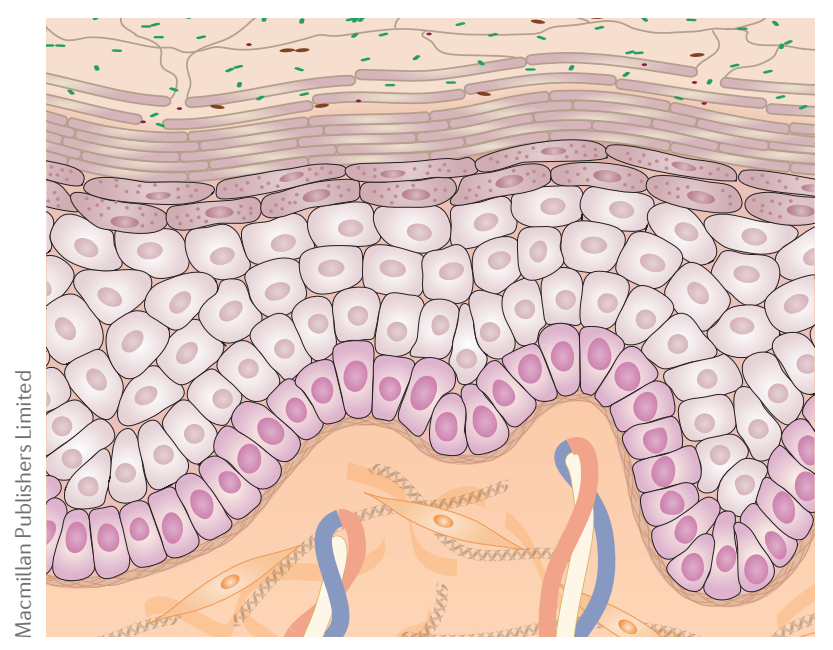

Numerous studies have previously implicated FLI1 in SSc pathogenesis. Asano et al. observe that FLI1 expression is lower in keratinocytes from patients with SSc than in those from healthy individuals, and that gene silencing of FLI1 in keratinocytes from healthy individuals results in an SSc-like gene expression profile. To further investigate the role of FLI1 in murine keratinocytes, the researchers crossed K14-Cre mice with Fli $1^{\mathrm{fl} / \mathrm{fl}}$ mice to generate mice in which FLI1 is knocked out in keratinocytes, some oesophageal epithelial cells and medullary thymic epithelial cells (mTECs), referred to here as K14-Cre.Fli1 ${ }^{\mathrm{f} / \mathrm{fl}}$ mice. "These mice exhibited an unexpectedly robust phenotype of autoimmunity as well as skin and oesophageal fibrosis. These phenotypes closely resemble human SSc phenotypes in various aspects," explains Asano.

The autoimmune phenotype in K14-Cre.Fli1 ${ }^{\mathrm{f} / \mathrm{fl}}$ mice included spontaneous development of interstitial lung disease (ILD). Using an adoptive T-cell transfer system, Asano and colleagues showed that the development of ILD, but not skin and oesophageal fibrosis, in K14-Cre.Fli ${ }^{\mathrm{f} / \mathrm{fl}}$ mice was dependent on autoreactive $\mathrm{T}$ cells. The researchers hypothesized that loss of FLI1 in mTECs could impair the expression of autoimmune regulator (AIRE). After confirming that murine mTECs strongly express FLI1 and that mTECs lacking FLI1 show reduced AIRE expression, Asano and colleagues used human keratinocytes to verify that FLI1 directly regulates AIRE transcription.

These results highlight the important role of FLI1 in autoimmune regulation, marking it out as a potentially therapeutic candidate. "Development of gene-modifying drugs that upregulate FLI1 expression might have beneficial effects in the treatment of patients," proposes Asano. "As skin keratinocytes and the epidermis are easily accessible by topical treatment this new approach might be advantageous."

Jessica McHugh

ORIGINAL ARTICLE Takahashi, T.et al. Epithelial

Fli1 deficiency drives systemic autoimmunity

and fibrosis: Possible roles in scleroderma.

J. Exp. Med. http://dx.doi.org/10.1084/ jem.20160247 (2017) 